\title{
Entropic Regularization Approach for Mathematical Programs with Equilibrium Constraints
}

\author{
Ş. Illker Birbil, Shu-Cherng Fang, Jiye Han
}

\begin{tabular}{|l|l|}
\hline \multicolumn{2}{|l|}{ ERIM REPORT SERIES RESEARCH IN MANAGEMENT } \\
\hline ERIM Report Series reference number & ERS-2002-71-LIS \\
\hline Publication & July 2002 \\
\hline Number of pages & 17 \\
\hline Email address corresponding author & sibirbil@few.eur.nl \\
\hline Address & Erasmus Research Institute of Management (ERIM) \\
& Rotterdam School of Management / Faculteit Bedrijfskunde \\
& Erasmus Universiteit Rotterdam \\
& P.O. Box 1738 \\
& 3000 DR Rotterdam, The Netherlands \\
& Phone: +31 104081182 \\
& Fax: $\quad+31104089640$ \\
& Email: info@erim.eur.nl \\
& Internet: $\quad$ www.erim.eur.nl \\
\hline
\end{tabular}

Bibliographic data and classifications of all the ERIM reports are also available on the ERIM website: www.erim.eur.nl 


\title{
ERASMUS RESEARCH INSTITUTE OF MANAGEMENT
}

\author{
REPORT SERIES \\ RESEARCH IN MANAGEMENT
}

\begin{tabular}{|c|c|c|}
\hline \multicolumn{3}{|c|}{ BIBLIOGRAPHIC DATA AND CLASSIFICATIONS } \\
\hline Abstract & \multicolumn{2}{|c|}{$\begin{array}{l}\text { A new smoothing approach based on entropic perturbation is proposed for solving mathematical } \\
\text { programs with equilibrium constraints. Some of the desirable properties of the smoothing function } \\
\text { are shown. The viability of the proposed approach is supported by a computational } \\
\text { study on a set of well-known test problems. }\end{array}$} \\
\hline \multirow{3}{*}{$\begin{array}{l}\text { Library of Congress } \\
\text { Classification } \\
\text { (LCC) }\end{array}$} & $5001-6182$ & Business \\
\hline & $5201-5982$ & Business Science \\
\hline & QA 1-939 & Mathematics \\
\hline \multirow{3}{*}{$\begin{array}{l}\text { Journal of Economic } \\
\text { Literature } \\
\text { (JEL) }\end{array}$} & M & Business Administration and Business Economics \\
\hline & $\begin{array}{l}\text { M } 11 \\
\text { R } 4\end{array}$ & $\begin{array}{l}\text { Production Management } \\
\text { Transportation Systems }\end{array}$ \\
\hline & $\begin{array}{l}\mathrm{C} 61 \\
\mathrm{C} 62\end{array}$ & $\begin{array}{l}\text { Programming models } \\
\text { Existence and Stability conditions of Equilibrium }\end{array}$ \\
\hline \multirow{3}{*}{$\begin{array}{l}\text { European Business Schools } \\
\text { Library Group } \\
\text { (EBSLG) }\end{array}$} & $85 \mathrm{~A}$ & Business General \\
\hline & $\begin{array}{l}260 \mathrm{~K} \\
240 \mathrm{~B}\end{array}$ & $\begin{array}{l}\text { Logistics } \\
\text { Information Systems Management }\end{array}$ \\
\hline & $250 \mathrm{~A}$ & Mathematics \\
\hline \multicolumn{3}{|c|}{ Gemeenschappelijke Onderwerpsontsluiting (GOO) } \\
\hline \multirow[t]{3}{*}{ Classification GOO } & 85.00 & Bedrijfskunde, Organisatiekunde: algemeen \\
\hline & $\begin{array}{l}85.34 \\
8520\end{array}$ & Logistiek management \\
\hline & 31.80 & Toepassingen van de wiskunde \\
\hline \multirow[t]{3}{*}{ Keywords GOO } & \multicolumn{2}{|c|}{ Bedrijfskunde / Bedrijsseconomie } \\
\hline & \multicolumn{2}{|c|}{ Bedrifsprocessen, logistiek, management informatiesystemen } \\
\hline & \multicolumn{2}{|c|}{ Wiskundige programmering } \\
\hline Free keywords & \multicolumn{2}{|c|}{ Mathematical programs with equilibrium constraints, smoothing approach, entropic regularization } \\
\hline
\end{tabular}




\title{
Entropic Regularization Approach for Mathematical Programs with Equilibrium Constraints
}

\author{
Ş. İlker Birbil * Shu-Cherng Fang ${ }^{\dagger} \quad$ Jiye Han ${ }^{\ddagger}$
}

July 24,2002

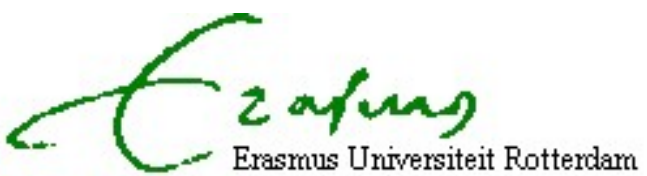

\begin{abstract}
A new smoothing approach based on entropic perturbation is proposed for solving mathematical programs with equilibrium constraints. Some of the desirable properties of the smoothing function are shown. The viability of the proposed approach is supported by a computational study on a set of well-known test problems.
\end{abstract}

Keywords Mathematical programs with equilibrium constraints, smoothing approach, entropic regularization.

\footnotetext{
${ }^{*}$ Erasmus Research Institute of Management (ERIM), Erasmus University, Postbus 1738, 3000 DR Rotterdam, The Netherlands. E-mail: sibirbilefew.eur.nl

${ }^{\dagger}$ Industrial Engineering and Operations Research, North Carolina State University, Raleigh, NC 26695-7906, USA. E-mail: fang@eos.ncsu.edu

${ }^{\ddagger}$ Institute of Applied Mathematics, Chinese Academy of Sciences, Beijing, P.R. China. E-mail: hjyyyf@ihw.com.cn
} 


\section{Introduction}

A mathematical program with equilibrium constraints (MPEC) is an optimization problem including essentially a set of parametric variational inequality or complementarity type constraints [Harker and Pang, 1988]. The "inner problem" corresponding to the variational inequality or complementarity type constraints, suggested the word "equilibrium", since in many engineering and economic applications, these constraints refer to a certain equilibrium phenomena. MPEC problems, which are known to be very difficult, arise in numerous areas such as network design, regional science, transportation planning, and game theory. We refer the interested reader to the extensive monograph by Luo et al. [1997].

There have been successful approaches to deal with this problem. One approach is based on general penalization techniques. This approach has been widely used and many good results have been reported [Ishizuka and Aiyoshi, 1992a,b]. Another approach is the application of heuristics. These heuristics are fairly general and do not require strict assumptions in handling MPEC problems [Suwansirikul et al., 1987, Friesz et al., 1992].

Outrata and Zowe [1995] reformulated the optimization problems with monotone variational inequalities. Their reformulation led to a nonsmooth Lipschitz optimization problem and hence benefited from the well-developed theory of nonsmooth optimization [Clarke, 1983]. In their paper, Outrata and Zowe successfully solved several academic problems and applied their approach to compute the well-known Stackelberg-Cournot-Nash equilibria [Murphy et al., 1982].

Recently, Kanzow and Jiang [1998] considered a continuation method for solving the monotone variational inequality problems. Their idea was based on solving a sequence of perturbed problems. The main tool for the perturbed problems was a specific smoothing function, which was also used by the authors in one of their earlier work [Kanzow, 1996]. They reported promising results on variational inequality and convex optimization problems.

Along this line, Facchinei et al. [1999] made use of a smoothing function from Kanzow and Jiang [1998] to solve MPEC problems. In addition to proposing an efficient algorithm, they showed its global convergence. Furthermore, they composed a set of test problems from the literature. Their numerical results on this set of problems demonstrated remarkable performance.

The primary aim of this paper is to exploit the application of a regularization approach to solve the MPEC problems. In particular, we are interested in using the entropy functions. The paper starts with the reformulation of the MPEC problem with nonsmooth constraints. To deal with the nonsmooth constraints, a regularization approach using the entropy function is applied. We validate the proposed approach by conducting a numerical study on a set of test problems collected from the literature. Our test results have shown that the proposed approach is able to converge to the best known results. In some cases, even finds better solutions than those reported in the literature.

The paper is organized as follows. Section 2 introduces an MPEC problem and a nonsmooth reformulation. Section 3 describes the proposed regularization approach with a discussion of its properties. The computational results over a set of test problems are reported in Section 4. The paper is concluded in Section 5. 


\section{Preliminaries}

We adopt the notation $\Re^{n}$ to denote the $n$-dimensional real vector space and $\|\cdot\|$ to denote the Euclidean norm in this space. In the sequel, the vectors in a real vector space are viewed as column vectors and the vector $\left(x^{T}, y^{T}, z^{T}, \lambda^{T}\right)^{T} \in \Re^{n} \times \Re^{m} \times \Re^{l} \times \Re^{l}$ is usually abbreviated by $(x, y, z, \lambda)$.

Let us first define a variational inequality problem with variables $x \in \Re^{n}$ and $y \in \Re^{m}$. Given a continuously differentiable function $F: \Re^{n+m} \rightarrow \Re^{m}$ and a set valued mapping

$$
C(x) \triangleq\left\{y \in \Re^{m}: g_{i}(x, y) \geq 0, i=1,2, \cdots, l\right\}
$$

with $g: \Re^{n+m} \rightarrow \Re^{l}$ being twice continuously differentiable and concave in the second variable, a variational inequality problem, $\operatorname{VI}(F, C)$ seeks for the solution set $S(x)$ such that $y \in S(x)$ if and only if $y \in C(x)$ and

$$
(v-y)^{T} F(x, y) \geq 0, \text { for all } v \in C(x) .
$$

Then, a typical MPEC becomes

$$
\begin{array}{lc}
\min & f(x, y) \\
\text { s.t. } & x \in X, \\
& y \in S(x),
\end{array}
$$

where $f: \Re^{n+m} \rightarrow \Re$ is a continuously differentiable function and $X$ is a nonempty set in $\Re^{n}$ [Luo et al., 1997].

Before proceeding to the subsequent analysis, let us first recall several definitions and results given by Harker and Pang [1990].

Definition 1 Given $x \in X$, let $F_{x}$ denote the vector-valued function $F(x, \cdot): C(x) \rightarrow \Re^{n}$, then $F_{x}$ is

(a) monotone if

$$
\left(y_{1}-y_{2}\right)^{T}\left(F_{x}\left(y_{1}\right)-F_{x}\left(y_{2}\right)\right) \geq 0 \text { for all } y_{1}, y_{2} \in C(x),
$$

(b) strongly monotone with modulus $\alpha>0$ if

$$
\left(y_{1}-y_{2}\right)^{T}\left(F_{x}\left(y_{1}\right)-F_{x}\left(y_{2}\right)\right) \geq \alpha\left\|y_{1}-y_{2}\right\|^{2} \text { for all } y_{1}, y_{2} \in C(x) .
$$

Theorem 1 [Harker and Pang, 1990, Corollary 3.2] Let $C(x)$ be a nonempty, closed convex set and $F(x, \cdot)$ be a strongly monotone and continuous mapping. Then the solution set of the variational inequalities $\mathrm{VI}(F, C)$ consists of a unique point.

Furthermore, define the set of active constraints as

$$
I(x, y) \triangleq\left\{i: g_{i}(x, y)=0\right\} .
$$

Then, the following definition facilitates an important assumption particularly for (KKT) optimality conditions.

Definition 2 A vector $y^{*}$ satisfies the linear independence constraint qualification (LICQ) if the gradients of the active inequality constraints are linearly independent. 
The following blanket assumptions similar to the ones in Outrata and Zowe [1995] are made throughout the paper.

(A1) $C(x) \neq \emptyset$ for all $x \in A$, where $A$ is an open bounded set in $\Re^{n}$ such that $X$ is contained in $A$.

(A2) $C(x)$ is uniformly compact on $A$, i.e., there exists an open bounded set $B \subset \Re^{m}$ such that $C(x) \subset B$ for all $x \in A$.

(A3) $F(x, \cdot)$ is strongly monotone.

(A4) $X$ is nonempty and compact in $\Re^{n}$.

(A5) At each $x \in X$ and $y \in S(x)$, the partial gradients $\nabla_{y} g_{i}(x, y), i \in I(x, y)$, satisfy (LICQ).

As a consequence of Theorem 1 , the above assumptions imply that for every $x$ in $X$, there exists a unique solution to $\mathrm{VI}(F, C)$. More specifically, assumption (A5) implies that every solution $y \in S(x)$ satisfies the KKT conditions

$$
\begin{gathered}
F(x, y)-\nabla_{y} g(x, y)^{T} \lambda=0, \\
g(x, y) \geq 0, \lambda \geq 0, \lambda^{T} g(x, y)=0,
\end{gathered}
$$

where the multiplier $\lambda \in \Re^{l}$ is uniquely determined. Moreover, (2) and (6) are equivalent [Harker and Pang, 1990, Proposition 2.2]. Therefore, we can reformulate the problem (MPEC) as a standard nonlinear complementarity constrained optimization problem

$$
\begin{array}{lc}
\min & f(x, y) \\
\text { s.t. } & x \in X, \\
& F(x, y)-\nabla_{y} g(x, y)^{T} \lambda=0, \\
& g(x, y) \geq 0, \lambda \geq 0, \lambda^{T} g(x, y)=0 .
\end{array}
$$

Though the problem (NLC) gives a smooth formulation, in general it does not satisfy any standard constraint qualification. In addition, the complementarity constraints are very difficult to handle [Falk and Liu, 1995]. Thus, following Facchinei et al. [1999], we consider a nonsmooth reformulation.

$$
\begin{array}{lc}
\min & f(x, y) \\
\text { s.t. } & x \in X, \\
& F(x, y)-\nabla_{y} g(x, y)^{T} \lambda=0, \\
& g(x, y)-z=0, \\
& \min (z, \lambda)=0,
\end{array}
$$

where $z \in \Re^{l}$ is added to simplify the notation and the "min" operator is applied componentwise to the vectors $z$ and $\lambda$. This problem can be further simplified to

$$
\begin{array}{lc}
\min & f(x, y) \\
\text { s.t. } & x \in X, \\
& H(x, y, z, \lambda)=0,
\end{array}
$$

where $H: \Re^{n+m+2 l} \rightarrow \Re^{m+2 l}$ is defined as

$$
H(x, y, z, \lambda) \triangleq\left(\begin{array}{c}
F(x, y)-\nabla_{y} g(x, y)^{T} \lambda \\
g(x, y)-z \\
\min (z, \lambda)
\end{array}\right) .
$$


The following proposition shows the equivalence of the problems (MPEC) and (NSM).

Theorem 2 [Facchinei et al., 1999] $\left(x^{*}, y^{*}\right)$ is a global (local) solution of the problem (MPEC) if and only if there exists a vector $\left(z^{*}, \lambda^{*}\right)$, which is a global (local) solution of the problem (NSM).

One major difficulty encountered in solving the problem (NSM) is the nondifferentiability of the "min" function. One approach uses the smoothing methods to approximate the "min" function [Chen and Mangasarian, 1995, Qi and Chen, 1995]. In particular, Kanzow [1996] used the following Chen-Harker-Kanzow-Smale (CHKS) smoothing function

$$
\phi_{\mu}(a, b)=a+b-\sqrt{(a-b)^{2}+4 \mu}
$$

where $\mu$ is the real parameter. Kanzow [1996] applied this aprroach successfully for solving linear complementarity problems, and then for solving monotone variational inequalities [Kanzow and Jiang, 1998]. Recently, Facchinei et al. [1999] benefited from this function in handling MPEC problems.

Here, we exploit the use of entropic regularization, since this particular approach has been shown to have useful properties [Fang and Wu, 1996, Li and Fang, 1997, Fang et al., 1997].

\section{Smoothing by Entropic Regularization}

The smoothing function $\phi_{p}: \Re^{2} \rightarrow \Re$ is defined as

$$
\phi_{p}(a, b) \triangleq-\frac{1}{p} \ln \left\{e^{-p a}+e^{-p b}\right\}
$$

where $p>0$ is the real parameter. Notice that, for each $p \neq 0, \phi_{p}(a, b)$ is a $C^{\infty}$ function. Furthermore, the following fundamental result shows that (10) defines a smooth approximation for the "min" function.

Lemma 1 Let $a, b \in \Re$ then for any $\epsilon>0$, there exists $P(\epsilon)>0$ such that

$$
\left|\phi_{p}(a, b)-\min (a, b)\right| \leq \epsilon \text { for all } p . \geq P(\epsilon)
$$

Proof. Assume that $\min (a, b)=a$, then

$$
\begin{aligned}
\phi_{p}(a, b) & =-\frac{1}{p} \ln \left\{e^{-p a}+e^{-p b}\right\} \\
& =-\frac{1}{p} \ln \left\{e^{-p a}\left[1+e^{-p(b-a)}\right]\right\} \\
& =-\frac{1}{p} \ln \left\{e^{-p a}\right\}-\frac{1}{p} \ln \left\{1+e^{-p(b-a)}\right\} \\
& =a-\frac{1}{p} \ln \left\{1+e^{-p(b-a)}\right\}
\end{aligned}
$$

By plugging this into (11) we have

$$
\left|a-\frac{1}{p} \ln \left\{1+e^{-p(b-a)}\right\}-a\right|=\frac{1}{p} \ln \left\{1+e^{-p(b-a)}\right\} \leq \frac{1}{p} \ln 2
$$


Therefore, the result follows with $P(\epsilon)=\frac{\ln 2}{\epsilon}$.

In nonlinear optimization, one of the desirable properties of a function is its convexity. Our next result shows that proposed function, $-\phi_{p}$ is convex.

Lemma 2 Given any $p>0, \phi_{p}$ is a concave function on $\Re^{2}$.

Proof. For any $u=\left(a_{1}, b_{1}\right), v=\left(a_{2}, b_{2}\right) \in \Re^{2}$ and $\alpha \in(0,1)$, define

$$
T_{p}(u, v) \triangleq \phi_{p}\left(\alpha a_{1}+(1-\alpha) a_{2}, \alpha b_{1}+(1-\alpha) b_{2}\right)
$$

Then we have

$$
\begin{aligned}
T_{p}(u, v) & =-\frac{1}{p} \ln \left\{e^{-\alpha p a_{1}} e^{-(1-\alpha) p a_{2}}+e^{-\alpha p b_{1}} e^{-(1-\alpha) p b_{2}}\right\} \\
& =-\frac{1}{p} \ln \left\{\left(e^{-p a_{1}}\right)^{\alpha}\left(e^{-p a_{2}}\right)^{1-\alpha}+\left(e^{-p b_{1}}\right)^{\alpha}\left(e^{-p b_{2}}\right)^{1-\alpha}\right\} .
\end{aligned}
$$

The Hölder Inequality [Kazarinoff, 1961, page 67] implies that

$$
\left(e^{-p a_{1}}\right)^{\alpha}\left(e^{-p a_{2}}\right)^{1-\alpha}+\left(e^{-p b_{1}}\right)^{\alpha}\left(e^{-p b_{2}}\right)^{1-\alpha} \leq\left(e^{-p a_{1}}+e^{-p b_{1}}\right)^{\alpha}\left(e^{-p a_{2}}+e^{-p b_{2}}\right)^{1-\alpha} .
$$

Consequently,

$$
\begin{aligned}
T_{p}(u, v) & \geq-\frac{\alpha}{p} \ln \left\{e^{-p a_{1}}+e^{-p b_{1}}\right\}-\frac{1-\alpha}{p} \ln \left\{e^{-p a_{2}}+e^{-p b_{2}}\right\} \\
& =\alpha \phi_{p}\left(a_{1}, b_{1}\right)+(1-\alpha) \phi_{p}\left(a_{2}, b_{2}\right) .
\end{aligned}
$$

Therefore, $\phi_{p}$ is a concave function on $\Re^{2}$.

Remark 1. Notice that a locally Lipschitz function is said to be regular if the directional derivative exists at all points and it is equal to its Clarke's directional derivative [Clarke, 1983]. As a consequence of Lemma 2, $\phi_{p}$ is a locally Lipschitz and regular function. This remark will be recalled in the sequel.

We now give a smooth reformulation of the problem (NSM). Let us define a nonlinear mapping $H_{p}: \Re^{n+m+2 l} \rightarrow \Re^{m+2 l}$,

$$
H_{p}(x, y, z, \lambda) \triangleq\left(\begin{array}{c}
F(x, y)-\nabla_{y} g(x, y)^{T} \lambda \\
g(x, y)-z \\
\Phi_{p}(z, \lambda)
\end{array}\right)
$$

where

$$
\Phi_{p}(z, \lambda) \triangleq\left(\phi_{p}\left(z_{1}, \lambda_{1}\right), \cdots, \phi_{p}\left(z_{l}, \lambda_{l}\right)\right)^{T} \in \Re^{l} .
$$

Thus, for each $p \in \Re$, we have

$$
\begin{array}{lc}
\min & f(x, y) \\
\text { s.t. } & x \in X, \\
& H_{p}(x, y, z, \lambda)=0 .
\end{array}
$$


Notice that as a result of Lemma 1, problem $\left(\mathrm{SM}_{p}\right)$ is a smooth perturbation of the problem (NSM). Let the feasible set of problem $\left(\mathrm{SM}_{p}\right)$ be $\mathcal{F}_{p} \in \Re^{n+m+2 l}$. To illustrate the formulation with entropic regularization, the following example is given.

Example 1 Monotone quasi-variational inequality problem [Harker, 1991].

$$
\begin{aligned}
& f(x, y)=\frac{1}{2}\left(\left(x_{1}-y_{1}\right)^{2}+\left(x_{2}-y_{2}\right)^{2}\right) \\
& X=[0,10] \times[0,10] \\
& F(x, y)=\left(\begin{array}{c}
-34+2 y_{1}+\frac{8}{3} y_{2} \\
-24.25+1.25 y_{1}+2 y_{2}
\end{array}\right) \\
& g_{1}(x, y)=-x_{2}-y_{1}+15 \\
& g_{2}(x, y)=-x_{1}-y_{2}+15
\end{aligned}
$$

The corresponding problems of (NLC) and $\left(\mathrm{SM}_{p}\right)$ can be derived as

$$
\begin{array}{lc}
\min & \frac{1}{2}\left(\left(x_{1}-y_{1}\right)^{2}+\left(x_{2}-y_{2}\right)^{2}\right) \\
\mathrm{s.t.} & 0 \leq x_{1}, x_{2} \leq 10 \\
& -34+2 y_{1}+\frac{8}{3} y_{2}+\lambda_{1}=0 \\
-24.25+1.25 y_{1}+2 y_{2}+\lambda_{2}=0 \\
-x_{2}-y_{1}+15 \geq 0 \\
-x_{1}-y_{2}+15 \geq 0 \\
\lambda_{1}, \lambda_{2} \geq 0 \\
\lambda_{1}\left(-x_{2}-y_{1}+15\right)=0 \\
\lambda_{2}\left(-x_{1}-y_{2}+15\right)=0
\end{array}
$$

and

$$
\begin{array}{lc}
\min & \frac{1}{2}\left(\left(x_{1}-y_{1}\right)^{2}+\left(x_{2}-y_{2}\right)^{2}\right) \\
\text { s.t. } & 0 \leq x_{1}, x_{2} \leq 10 \\
& -34+2 y_{1}+\frac{8}{3} y_{2}+\lambda_{1}=0 \\
-24.25+1.25 y_{1}+2 y_{2}+\lambda_{2}=0 \\
-x_{2}-y_{1}+15-z_{1}=0 \\
-x_{1}-y_{2}+15-z_{2}=0 \\
-\frac{1}{p} \ln \left\{e^{-p z_{1}}+e^{-p \lambda_{1}}\right\}=0 \\
-\frac{1}{p} \ln \left\{e^{-p z_{2}}+e^{-p \lambda_{2}}\right\}=0
\end{array}
$$

respectively.

Theorem 3 Given any $p \neq 0$ and $(x, y, z, \lambda) \in \mathcal{F}_{p}$, the Jacobian of $H_{p}$ with respect to the variables $(y, z, \lambda)$ is nonsingular.

Proof. Since $\phi_{p}, p \neq 0$, is continuously differentiable on $\Re^{2}$, the operator $H_{p}$ is also continuously differentiable. Hence, its Jacobian is given by

$$
\nabla H_{p}(x, y, z, \lambda)=\left(\begin{array}{ccc}
Q & 0 & -A^{T} \\
A & -I_{l} & 0 \\
0 & D_{1} & D_{2}
\end{array}\right)
$$


where

$$
\begin{aligned}
Q & \triangleq \nabla_{y} F(x, y)-\sum_{i \in I} \lambda_{i} \nabla_{y}^{2} g_{i}(x, y) \\
A & \triangleq \nabla_{y} g(x, y) \\
D_{1} & \triangleq \operatorname{diag}\left(\frac{\partial \phi_{p}}{\partial z_{1}}\left(z_{1}, \lambda_{1}\right), \cdots, \frac{\partial \phi_{p}}{\partial z_{l}}\left(z_{l}, \lambda_{l}\right)\right) \\
D_{2} & \triangleq \operatorname{diag}\left(\frac{\partial \phi_{p}}{\partial \lambda_{1}}\left(z_{1}, \lambda_{1}\right), \cdots, \frac{\partial \phi_{p}}{\partial \lambda_{l}}\left(z_{l}, \lambda_{l}\right)\right)
\end{aligned}
$$

and $I_{l}$ is the $l$-dimensional identity matrix. Notice that since $F(x, \cdot)$ is strongly monotone, $\nabla_{y} F(x, \cdot)$ is positive definite. Moreover since all functions $g_{i}(x, \cdot)$ are concave, their Hessian matrices $\nabla_{y}^{2} g_{i}(x, \cdot)$ are negative semidefinite for $i \in I$. Therefore, the matrix $Q$ is positive definite. For all $i=1,2, \cdots, l$, since

$$
\begin{aligned}
& \frac{\partial \phi_{p}\left(z_{i}, \lambda_{i}\right)}{\partial z_{i}}=\frac{e^{-p z_{i}}}{e^{-p z_{i}}+e^{-p \lambda_{i}}} \in(0,1) \text { and } \\
& \frac{\partial \phi_{p}\left(z_{i}, \lambda_{i}\right)}{\partial \lambda_{i}}=\frac{e^{-p \lambda_{i}}}{e^{-p z_{i}}+e^{-p \lambda_{i}}} \in(0,1),
\end{aligned}
$$

the diagonal matrices $D_{1}$ and $D_{2}$ are positive definite. To show the nonsingularity of $\nabla H_{p}$, assume that $\nabla H_{p} u=0$ for some vector $u=\left(u^{(1)}, u^{(2)}, u^{(3)}\right) \in \Re^{m} \times \Re^{l} \times \Re^{l}$. Then

$$
\begin{aligned}
Q u^{(1)}-A^{T} u^{(3)} & =0 \\
A u^{(1)}-u^{(2)} & =0 \\
D_{1} u^{(2)}+D_{2} u^{(3)} & =0
\end{aligned}
$$

Substituting $u^{(2)}$ from (36) into (37) leads to

$$
u^{(3)}=-D_{2}^{-1} D_{1} A u^{(1)} .
$$

Plugging $u^{(3)}$ into (35) yields

$$
Q u^{(1)}+A^{T} D_{2}^{-1} D_{1} A u^{(1)}=\left(Q+A^{T} D_{2}^{-1} D_{1} A\right) u^{(1)}=0 .
$$

Notice that $D_{2}^{-1} D_{1}$ and $Q$ are positive definite, hence $u^{(1)}=0$. From (37) and (38), we have $u^{(3)}=0$ and $u^{(2)}=0$. Therefore, $\nabla H_{p}$ is nonsingular.

Remark 2. A vector function is said to be locally Lipschitz and regular if each of its components is locally Lipschitz and regular. We have already mentioned in Remark 1 that $\phi_{p}$ satisfies this property. Since all the remaining components of $H_{p}$ are continuously differentiable, we can conclude that $H_{p}$ is a locally Lipschitz and regular function.

Note that locally Lipschitz and regular functions are useful for the application of implicit function theorem, which leads to the convergence properties of the nonlinear programming algorithms [Outrata and Zowe, 1995, Kanzow, 1996]. 


\section{Numerical Results}

Note that specific algorithms can be developed for the entropic regularization approach. However, we are interested in investigating the performance of the proposed approach by using a publicly available software. In general, people tend to benefit from commercial or freeware software instead of creating their own tool. In this respect, we decided to use NEOS server, which provides access to many optimization solvers through the internet. Especially, the Kestrel interface enables the remote solution of optimization problems within the AMPL and GAMS modeling languages [Dolan and Munson, 2001]. We used AMPL as our modeling language, which is also available through the internet [AMPL, 2001].

SNOPT is one of the recently updated solvers on the NEOS server. It employs a sparse sequential quadratic programming algorithm with (quasi) Newton approximations. In particular, SNOPT allows the nonlinear constraints to be violated (if necessary) and minimizes the sum of such violations [Gill et al., 2000]. Therefore, we selected it to be the solver for our numerical study. We remark that we did not seek for optimal parameter settings for SNOPT but used the default parameters.

Recently, Facchinei et al. [1999] created a set of frequently cited problems, and reported their results. They developed their own algorithms and conducted computations by using a commercial package. We took advantage of their work and applied our approach to this set of problems. For fair comparision, we solved all the test problems by using both their smoothing approach and the proposed approach.

In Facchinei et al. [1999], the perturbation function (9) is used. We set the parameter, $\mu$ to be the initial choice of $1.0 \mathrm{e}-4$, since they reported that their approach did not seem to depend critically on this choice. However, our limited experience showed that reducing the value of this parameter increases the precision at the cost of excessive iterations. Thus, we did not adjust this parameter unless we observed radical differences between our results and the results that they reported. We abbreviate their approach by FSA and entropic regularization approach by ERA in the subsequent discussion.

In Table 1, the first column numbers the test problems in the same order as Facchinei et al. reported. But, we initiated each problem with more different starting points than they did. Thus, the second column includes their starting points as well as additional starting points (which produced interesting results). Columns three and six give the best objective function values $\left(f^{*}\right)$ reported by SNOPT. All the test problems are minimization problems, hence the smaller objective function values mean the better solutions are obtained. Corresponding to these objective function values, columns four and seven give the optimum solutions.

Notice that for both FSA and ERA, the required computational work at each iteration is about the same. Therefore, the number of iterations given in columns five and eight shows how quickly the solver converges to the reported objective function value. The last column gives the parameter $p$ for entropic regularization approach. In each problem we initially set this parameter to $1.0 \mathrm{e} 4$.

Observe that, except for problem 11, FSA gave the same results as reported by Facchinei et al. [1999]. Note that, ERA demonstrated a remarkable performance and was able to solve each test problem in a reasonable number of iterations. For problems 1 to 5, FSA and ERA produced the same results. However, for problem 6, ERA perfomed exceptionally well. In fact, this result has never been reported in any work that we know of [Outrata and Zowe, 1995, 
Riesz et al., 1990]. To make sure, we reduced the parameter of FSA down to 1.0e-12, but the result did not change. For problem 7, although ERA was able to converge to the vicinity of the reported optimum solution, the precision of the objective function value is not as good as FSA.

For problems 8.1 to 10 both approaches were able to converge to the same optimum solutions. For problem 11, we reduced the parameter of FSA down to 1.0e-12. The optimum value was reached with the first starting point, but FSA failed with the remaining two starting points. ERA did not observe any difficulty in converging to the optimal solution with the first two starting points, but stuck in the local optimum with the last starting point.

One more observation worth mentioning. ERA slows down when an initial starting point is selected to be the 0 vector and the parameter $p$ is not large enough (for problems 2,9 and $11)$. Because in this case, $\phi_{p}(0,0)=-\frac{\ln 2}{p}$, and hence excessive iterations are spent to satisfy the violated equality constraint.

In our computational study, we observed that overflow problems may arise due to the exponential function in (10). We experienced this phenomenon for problems 6,7, 9 and 10. However, except problem 7, the results were not affected. Consequently, for this particular set of problems, we did not tackle this issue explicitly.

To handle the overflow problem, the smoothing function (10) can be slightly modified without changing its properties. For example, when we compute the exponential function $e^{-p a}$ with $p a<0$ and very large $|p a|$, the overflow problem occurs in the smoothing function. This problem can be avoided by using the following equality:

$$
\phi_{p}(a, b) \triangleq-\frac{1}{p} \ln \left\{e^{-p a+p c}+e^{-p b+p c}\right\}+c
$$

where $c$ is any value satisfying $c \leq \min (a, b)$. Notice that both $-p a+p c \leq 0$ and $-p b+p c \leq 0$, hence the overflow problem is handled effectively. 


\begin{tabular}{|c|c|c|c|c|c|c|c|c|}
\hline \multirow{2}{*}{ No. } & & \multicolumn{3}{|c|}{ FSA } & \multicolumn{4}{|c|}{ ERA } \\
\hline & Start & $f^{*}$ & $x^{*}$ & It. & $f^{*}$ & $x^{*}$ & It. & $\mathrm{p}$ \\
\hline \multirow{2}{*}{1} & 0.0 & 3.2078 & 4.0605 & 26 & 3.2077 & 4.0604 & 32 & \multirow{2}{*}{$1.0 \mathrm{e} 4$} \\
\hline & 10.0 & 3.2078 & 4.0605 & 29 & 3.2077 & 4.0604 & 29 & \\
\hline \multirow{2}{*}{2} & 0.0 & 3.4494 & 5.1536 & 28 & 3.4494 & 5.1536 & 33 & \multirow{2}{*}{$1.0 \mathrm{e} 4$} \\
\hline & 10.0 & 3.4494 & 5.1536 & 27 & 3.4494 & 5.1536 & 19 & \\
\hline \multirow[b]{2}{*}{3} & 0.0 & 4.6042 & 2.3894 & 21 & 4.6042 & 2.3894 & 29 & \multirow{2}{*}{$1.0 \mathrm{e} 4$} \\
\hline & 10.0 & 4.6042 & 2.3894 & 21 & 4.6041 & 2.3894 & 28 & \\
\hline \multirow{2}{*}{4} & 0.0 & 6.5926 & 1.3731 & 30 & 6.5926 & 1.3731 & 23 & \multirow{2}{*}{$1.0 \mathrm{e} 4$} \\
\hline & 10.0 & 6.5926 & 1.3731 & 22 & 6.5926 & 1.3731 & 28 & \\
\hline \multirow{2}{*}{5} & $(0.0,0.0)$ & -0.9999 & $(0.5010,0.5010)$ & 15 & -0.9999 & $(0.5010,0.5010)$ & 13 & \multirow{2}{*}{$1.0 \mathrm{e} 4$} \\
\hline & $(2.0,2.0)$ & -0.9999 & $(0.5010,0.5010)$ & 19 & -0.9999 & $(0.5010,0.5010)$ & 21 & \\
\hline \multirow{3}{*}{6} & 0.0 & -3266.6666 & 93.3333 & 7 & -4512.5000 & 95.0000 & 10 & \multirow{3}{*}{$1.0 \mathrm{e} 2$} \\
\hline & 100.0 & -3266.6666 & 93.3333 & 6 & -4512.5000 & 95.0000 & 6 & \\
\hline & 200.0 & -3266.6666 & 93.3333 & 6 & -4512.5000 & 95.0000 & 6 & \\
\hline \multirow{2}{*}{7} & $(25.0,25.0)$ & 4.9996 & $(25.0012,30.0001)$ & 44 & 5.0151 & $(24.9978,30.01812)$ & 35 & \multirow{2}{*}{$1.0 \mathrm{e} 2$} \\
\hline & $(50.0,50.0)$ & 4.9996 & $(25.0012,30.0001)$ & 43 & 5.0151 & $(24.9978,30.01812)$ & 41 & \\
\hline \multirow{2}{*}{8.1} & 0.0 & -343.3452 & 55.5513 & 19 & -343.3452 & 55.5513 & 19 & \multirow{2}{*}{$1.0 \mathrm{e} 4$} \\
\hline & 150.0 & -343.3452 & 55.5513 & 23 & -343.3452 & 55.5513 & 23 & \\
\hline \multirow{2}{*}{8.2} & 0.0 & -203.1550 & 42.5383 & 25 & -203.1550 & 42.5383 & 24 & \\
\hline & 150.0 & -203.1550 & 42.5383 & 22 & -203.1550 & 42.5383 & 22 & $1.0 \mathrm{e} 4$ \\
\hline & 0.0 & -68.1356 & 24.1451 & 22 & -68.1356 & 24.1450 & 20 & \\
\hline 8.3 & 150.0 & -68.1356 & 24.1451 & 22 & -68.1356 & 24.1450 & 21 & $1.0 \mathrm{e} 4$ \\
\hline 81 & 0.0 & -19.1540 & 12.3726 & 22 & -19.1540 & 12.3726 & 20 & \\
\hline 0.4 & 150.0 & -19.1540 & 12.3726 & 21 & -19.1540 & 12.3726 & 21 & $1.0 \mathrm{e} 4$ \\
\hline 85 & 0.0 & -3.1611 & 4.7536 & 22 & -3.1611 & 4.7536 & 20 & $10 \mathrm{e} 4$ \\
\hline 0.5 & 150.0 & -3.1611 & 4.7536 & 21 & -3.1611 & 4.7536 & 21 & 1.004 \\
\hline & 0.0 & -346.8931 & 50.0000 & 16 & -346.8932 & 50.0000 & 21 & \\
\hline 8.6 & 50.0 & -346.8931 & 50.0000 & 17 & -346.8931 & 50.0000 & 17 & $1.0 \mathrm{e} 4$ \\
\hline & 0.0 & -224.0371 & 39.7915 & 21 & -224.0371 & 39.7913 & 25 & 1004 \\
\hline 8.1 & 40.0 & -224.0371 & 39.7915 & 17 & -224.0372 & 39.7913 & 15 & $1.0 \mathrm{e} 4$ \\
\hline 88 & 0.0 & -80.7859 & 24.2571 & 21 & -80.7859 & 24.2571 & 29 & $10 \mathrm{e} 4$ \\
\hline 8.8 & 30.0 & -80.7859 & 24.2571 & 19 & -80.7859 & 24.2571 & 19 & $1.0 \mathrm{e} 4$ \\
\hline 80 & 0.0 & -22.8371 & 13.0196 & 21 & -22.8371 & 13.0196 & 20 & $10 \bigcirc 4$ \\
\hline 8.9 & 30.0 & -22.8371 & 13.0196 & 19 & -22.8371 & 13.0196 & 19 & $1.0 \mathrm{e} 4$ \\
\hline 810 & 0.0 & -5.3491 & 6.0023 & 20 & -5.3491 & 6.0023 & 20 & $10 \mathrm{e} 4$ \\
\hline 0.10 & 20.0 & -5.3491 & 6.0023 & 19 & -5.3491 & 6.0023 & 19 & 1.004 \\
\hline & $(0.0,0.0)$ & $4.19 \mathrm{e}-12$ & $(5.0000,9.0000)$ & 16 & $6.34 \mathrm{e}-11$ & $(5.0000,9.0000)$ & 15 & \\
\hline 9 & $(5.0,5.0)$ & $4.20 \mathrm{e}-13$ & $(5.0000,8.9999)$ & 11 & $7.20 \mathrm{e}-14$ & $(5.0000,8.9999)$ & 11 & $1.0 \mathrm{e} 2$ \\
\hline & $(10.0,10.0)$ & $2.23 \mathrm{e}-10$ & $(9.0004,5.9995)$ & 27 & $4.50 \mathrm{e}-11$ & $(9.0976,5.9023)$ & 31 & \\
\hline & $(10.0,0.0)$ & $2.38 \mathrm{e}-10$ & $(9.0004,5.9995)$ & 24 & $8.32 \mathrm{e}-11$ & $(9.0937,5.9062)$ & 32 & \\
\hline & $(0.0,10.0)$ & $6.19 \mathrm{e}-12$ & $(5.0000,9.0000)$ & 18 & $6.19 \mathrm{e}-12$ & $(5.0000,9.0000)$ & 19 & \\
\hline & $(0.0,0.0,0.0,0.0)$ & -6600.0000 & $(7.0,3.0,12.0,18.0)$ & 83 & -6600.0000 & $(7.0,3.0,12.0,18.0)$ & 59 & \\
\hline & $(0.0,5.0,0.0,20.0)$ & -6600.0000 & $(7.0,3.0,12.0,18.0)$ & 81 & -6600.0000 & $(7.0,3.0,12.0,18.0)$ & 68 & \\
\hline 10 & $(5.0,0.0,15.0,10.0)$ & -6600.0000 & $(7.0,3.0,12.0,18.0)$ & 76 & -6600.0000 & $(7.0,3.0,12.0,18.0)$ & 62 & $1.0 \mathrm{e} 2$ \\
\hline & $(5.0,5.0,15.0,15.0)$ & -6600.0000 & $(7.0,3.0,12.0,18.0)$ & 79 & -6600.0000 & $(7.0,3.0,12.0,18.0)$ & 66 & \\
\hline & $(10.0,5.0,15.0,10.0)$ & -6600.0000 & $(7.0,3.0,12.0,18.0)$ & 53 & -6600.0000 & $(7.0,3.0,12.0,18.0)$ & 44 & \\
\hline & $(0.0,0.0)$ & -12.6787 & $(0.0000,2.0000)$ & 40 & -12.6787 & $(0.0000,2.0000)$ & 53 & \\
\hline 11 & $(0.0,2.0)$ & -8.6364 & $(1.8003,0.0371)$ & 20 & -12.6787 & $(0.0000,2.0000)$ & 21 & $1.0 \mathrm{e} 1$ \\
\hline & $(2.0,0.0)$ & -8.6364 & $(1.8003,0.0371)$ & 23 & -10.3567 & $(2.0000,0.0000)$ & 38 & \\
\hline
\end{tabular}

Table 1: Comparison of the entropic regularization approach with Facchinei et al. [1999] 


\section{Conclusion}

We have proposed a regularization approach using an entropy function for solving mathematical programs with equilibrium constraints. The proposed approach has been shown that it can be easily implemented. Instead of developing any specific software, we have used a publicly available solver with default parameters to compare the proposed approach with recently reported results of Facchinei et al. [1999] over a set of test problems. Our numerical results have shown that proposed approach has a very promising performance and is able to converge to the best known results. Moreover, the proposed approach has even found better solutions than those reported in the literature.

\section{References}

AMPL. AMPL home page, 2001. http://www.ampl.com.

C. Chen and O.L. Mangasarian. Smoothing methods for convex inequalities and linear complementarity problems. Mathematical Programming, 71:51-69, 1995.

F.H. Clarke. Optimization and Nonsmooth Analysis. Wiley, New York, 1983.

E.D. Dolan and T.S. Munson. The Kestrel interface to the NEOS server, 2001. http://wwwneos.mcs.anl.gov/neos/ftp/kestrel.ps.

F. Facchinei, H. Jiang, and L. Qi. A smoothing method for mathematical programs with equilibrium constraints. Mathematical Programming, 85:107-134, 1999.

J.E. Falk and J. Liu. On bilevel programming, part I: general nonlinear cases. Mathematical Programming, 48:47-72, 1995.

S.-C. Fang, J.R. Rajasekera, and H.-S. Tsao. Entropy Optimization and Mathematical Programming. Kluwer Academic Publishers, Norwell, 1997.

S.-C. Fang and S.-Y. Wu. Solving min-max problems and linear semi-infinite programs. Comput. Math. Applicat., 32:87-93, 1996.

T.L. Friesz, H.-J. Cho, N.J. Mehta, R.L. Tobin, and G. Anandalingam. A simulated annealing approach to the network design problem with variational inequality constraints. Transportation Science, 26:18-26, 1992.

P.E. Gill, W. Murray, M.A. Saunders, A. Drud, and E. Kalvelagen. GAMS/SNOPT: An SQP algorithm for large-scale constrained optimization, 2000. http://www.gams.com/docs/solver/snopt.pdf.

P.T. Harker. Generalized Nash games and quasi-variational inequalities. Eur. J. Oper. Res., 54: 81-94, 1991.

P.T. Harker and J.-S. Pang. On the existence of optimal solutions to mathematical program with equilibrium constraints. Operations Research Letters, 7:61-64, 1988. 
P.T. Harker and J.-S. Pang. Finite-dimensional and variational inequality and nonlinear complementarity problems: A survey of theory, algorithms, and applications. Mathematical Programming, 48:161-220, 1990.

Y. Ishizuka and E. Aiyoshi. Double penalty method for bilevel optimization problems. In G. Anandalingam and T.L. Friesz, editors, Hierarchical Optimization. Annals of Operations Research, volume 34, pages 73-88. Baltzer Science Publishers, 1992a.

Y. Ishizuka and E. Aiyoshi. Regularizations for two-level optimization problems. In Advances in Optimization, pages 239-255. Springer Verlag, 1992b.

C. Kanzow. Some noninterior continuation methods for linear complementarity problems. SIAM J. Matrix Anal. Appl., 17:851-868, 1996.

C. Kanzow and H. Jiang. A continuation method for (strongly) monotone variational inequalities. Mathematical Programming, 89:103-125, 1998.

N.D. Kazarinoff. Analytic Inequalities. Holt, Rinehart and Winston, New York, 1961.

X.-S. Li and S.-C. Fang. On the entropic regularization method for solving min-max problems with applications. Mathematical Methods of Operations Research, 46:119-130, 1997.

Z.-Q. Luo, J.-S. Pang, and D. Ralph. Mathematical Programs with Equilibrium Constraints. Cambridge University Press, Cambridge, 1997.

F.H. Murphy, H.D. Sherali, and A.L. Soyster. A mathematical programming approach for determining oligopolistic market equilibrium. Mathematical Programming, 24:92-106, 1982.

J. Outrata and J. Zowe. A numerical approach to optimization problems with variational inequality constraints. Mathematical Programming, 68:105-130, 1995.

L. Qi and X. Chen. A globally convergent successive approximation methods for nonsmooth equations. SIAM J. Control Optim., 33:402-418, 1995.

T.L. Riesz, R.L. Tobin, H.-J. Chao, and N.J. Mehta. Sensitivity analysis based heuristic algorithms for mathematical programs with variational inequalities. Mathematical Programming, 48:265-284, 1990.

C. Suwansirikul, T.L. Friesz, and R.L. Robin. Equilibrium decomposed optimization: A heuristic for the continuous equilibrium network design problem. Transportation Science, 21:254-263, 1987. 


\title{
Publications in the Report Series Research* in Management
}

\author{
ERIM Research Program: "Business Processes, Logistics and Information Systems"
}

\section{2}

The importance of sociality for understanding knowledge sharing processes in organizational contexts Niels-Ingvar Boer, Peter J. van Baalen \& Kuldeep Kumar ERS-2002-05-LIS

Crew Rostering for the High Speed Train

Ramon M. Lentink, Michiel A. Odijk \& Erwin van Rijn

ERS-2002-07-LIS

Equivalent Results in Minimax Theory

J.B.G. Frenk, G. Kassay \& J. Kolumbán

ERS-2002-08-LIS

An Introduction to Paradigm

Saskia C. van der Made-Potuijt \& Arie de Bruin

ERS-2002-09-LIS

Airline Revenue Management: An Overview of OR Techniques 1982-2001

Kevin Pak \& Nanda Piersma

ERS-2002-12-LIS

Quick Response Practices at the Warehouse of Ankor

R. Dekker, M.B.M. de Koster, H. Van Kalleveen \& K.J. Roodbergen

ERS-2002-19-LIS

Harnessing Intellectual Resources in a Collaborative Context to create value Sajda Qureshi, Vlatka Hlupic, Gert-Jan de Vreede, Robert O. Briggs \& Jay Nunamaker ERS-2002-28-LIS

Version Spaces and Generalized Monotone Boolean Functions

Jan C. Bioch \& Toshihide Ibaraki

ERS-2002-34-LIS

Periodic Review, Push Inventory Policies for Remanufacturing

B. Mahadevan, David F. Pyke, Moritz Fleischman

ERS-2002-35-LIS

Modular Decomposition of Boolean Functions

Jan C. Bioch

ERS-2002-37-LIS

Classification Trees for Problems with Monotonicity Constraints

R. Potharst \& A.J. Feelders

ERS-2002-45-LIS

A complete overview of the ERIM Report Series Research in Management: http://www.ers.erim.eur.nl

ERIM Research Programs:

LIS Business Processes, Logistics and Information Systems

ORG Organizing for Performance

MKT Marketing

F\&A Finance and Accounting

STR Strategy and Entrepreneurship 
Allocation of Railway Rolling Stock for Passenger Trains

Erwin Abbink, Bianca van den Berg, Leo Kroon \& Marc Salomon

ERS-2002-47-LIS

Monotone Decision Trees and Noisy Data

Jan C. Bioch and Viara Popova

ERS-2002-53-LIS

Business Modeling Framework For Personalization In Mobile Business Services: a Case and Sociological Analysis L-F Pau, Jeroen Dits

ERS-2002-56-LIS

Polynomial time algorithms for some multi-level lot-sizing problems with production capacities

Stan van Hoesel, H. Edwin Romeijn, Dolores Romero Morales, Albert P.M. Wagelmans

ERS-2002-59-LIS

A Note on Ending Inventory Valuation in Multiperiod Production Scheduling

Wilco van den Heuvel, Alfred P.M. Wagelmans

ERS-2002-63-LIS

Determining The Optimal Order Picking Batch Size In Single Aisle Warehouses

Tho Le-Duc and René B.M. de Koster

ERS-2002-64-LIS

Solving Variational Inequalities Defined on A Domain with Infinitely Many Linear Constraints

Shu-Cherng Fang, Soonyi Wu, Ş. Illker Birbil

ERS-2002-70-LIS

Entropic Regularization Approach for Mathematical Programs with Equilibrium Constraints

Ş. Illker Birbil, Shu-Cherng Fang, Jiye Han

ERS-2002-71-LIS

On the Finite Termination of An Entropy Function Based Smoothing Newton Method for Vertical Linear

Complementarity Problems

Shu-Cherng Fang, Jiye Han, Zhenghai Huang, Ş. İlker Birbil

ERS-2002-72-LIS

The Role Of Product Quality Information, Market State Information And Transaction Costs In Electronic Auctions

Otto Koppius and Eric van Heck

ERS-2002-73-LIS

Shunting of Passenger Train Units in a Railway Station

Richard Freling, Ramon M. Lentink, Leo G. Kroon, Dennis Huisman

ERS-2002-74-LIS

Inventory strategies for systems with fast remanufacturing

Ruud Teunter, Erwin van der Laan, Dimitrios Vlachosz

ERS-2002-77-LIS

A Business Evaluation Of The Next IPv6 Protocol In Fixed And Mobile Communication Services:

An Analytical Study And Calculation

L-F Pau

ERS-2002-78-LIS 